\title{
A Method for Retrieving Mean Horizontal Wind Profiles from Single-Doppler Radar Observations Contaminated by Aliasing
}

\author{
JIDONG GAO \\ Center for Analysis and Prediction of Storms, University of Oklahoma, Norman, Oklahoma \\ KeLVIN K. DROegemeier \\ Center for Analysis and Prediction of Storms and School of Meteorology, University of Oklahoma, Norman, Oklahoma \\ JIANDONG GONG \\ Cooperative Institute for Mesoscale Meteorological Studies, University of Oklahoma, Norman, Oklahoma \\ QIN XU \\ Cooperative Institute for Mesoscale Meteorological Studies, University of Oklahoma, and National Severe Storms Laboratory, \\ Norman, Oklahoma
}

(Manuscript received 24 June 2003, in final form 4 December 2003)

\begin{abstract}
The velocity-azimuth display (VAD) technique was designed to estimate the areal mean vertical profile of the horizontal wind above a ground-based Doppler radar. The method uses radial velocity observations under the assumption of a linear wind field, though it encounters difficulty when the observations are contaminated by velocity ambiguities, large noise, and when viable data exist only over a restricted azimuthal range. The method suggested in this paper uses gradients of radial velocity, rather than only the velocity itself, to derive wind profiles and thus is termed the gradient velocity-azimuth display (GVAD) technique.

Both the VAD and GVAD methods are tested first on simulated data to examine their sensitivity to different type of errors in radial velocity. The retrieved mean wind profiles are shown to be insensitive to random errors in radial velocity, even at large amplitude. However, the VAD method is very sensitive to systematic errors caused by velocity ambiguities. The experiments indicate that if only $3 \%$ of a full-volume scan of radial wind data is contaminated by aliasing errors, the relative rms error in the mean wind profile retrieved by VAD can reach $50 \%$. In contrast, GVAD is very robust to such errors.

Application of GVAD to Weather Surveillance Radar-1988 Doppler (WSR-88D) data collected during the 3 May 1999 tornado outbreak show that it has the ability to obtain accurate wind profiles even when the observations contain large errors caused by velocity ambiguities and random noise.
\end{abstract}

\section{Introduction}

The velocity-azimuth display (VAD) technique, pioneered by Lhermitte and Atlas (1961) and Browning and Wexler (1968), has been used to obtain the areal mean vertical profile of the horizontal wind above a ground-based Doppler radar. Under the assumption that the wind field at a given altitude is uniform across the region being scanned by the radar, the VAD method involves fitting a sinusoidal curve through radial wind data to yield the mean radial Doppler velocity for a fixed

Corresponding author address: Dr. Jidong Gao, Center for Analysis and Prediction of Storms, Sarkeys Energy Center, Suite 1110, 100 East Boyd, Norman, OK 73019.

E-mail: jdgao@ou.edu elevation as a function of azimuth. Improved versions of the VAD technique, for additional extraction of divergence and vertical velocity, include the extended VAD (Srivastava et al. 1986; Matejka and Srivastava 1991) and concurrent extended VAD (Matejka 1993).

Instead of processing data along a circle for a fixed range in $\mathrm{VAD}$, one also can process simultaneously all available data at a certain altitude. This so-called volume velocity processing (VVP) technique was introduced by Waldteufel and Corbin (1979) and applied by Koscielny et al. (1982). Using a linear wind assumption, the parameters of the wind field can be calculated from all points within a layer centered at a given altitude through a multiparameter linear fit. The VVP technique typically is applied to thin layers of data at successive altitudes to obtain a vertical profile of the horizontal wind. The 
stability and robustness of the VAD and VVP regressions were explored by Boccippio (1995).

VAD (or VVP) winds have practical application in at least three areas. First, for large-scale data assimilation, a single sounding typically is used to represent atmospheric conditions over a fairly large region. The spacing of rawinsonde stations is about $400 \mathrm{~km}$ over the continental United States. VAD winds, however, represent a mean over an area and smooth out many of the transient or local effects such as turbulent motions and small-scale terrain-induced features so that only the larger mesoscale and synoptic-scale features of the wind field remain. Currently, more than 200 operational NextGeneration Weather Radars (NEXRADs) and Terminal Doppler Weather Radars (TDWRs) are distributed across the united states, and the number of routine radiosonde sites has declined gradually to about 70 . Therefore, wind profiles produced from operational Doppler radars routinely provide over $50 \%$ greater horizontal resolution at much more frequent intervals (every 510-min update). Such data have tremendous value for large-scale synoptic four-dimensional data assimilation and for other meteorological applications (Michelson and Seaman 2000), although no information about temperature and pressure is available directly. VAD (or VVP) wind profiles also are very useful to operational National Weather Service (NWS) forecasters for monitoring the height of low-level jets, anticipating thunderstorm type and severity, issuing high-wind warnings, monitoring wave passages, monitoring strong upper-level winds, and depicting frontal boundaries aloft (Serafin and Wilson 2000).

Secondly, VAD also can provide background information for full three-dimensional wind retrievals from Doppler radar radial velocity data. During the past several years, many velocity retrieval algorithms have been developed and tested with data from several types of radars (e.g., Qiu and Xu 1992; Xu and Qiu 1994, 1995; Xu et al. 1994; Shapiro et al. 1995, 2003; Sun and Crook 1997, 1998; Sun et al. 1991; Weygandt et al. 1995, 2002; Gao et al. 1999, 2001). Application of these techniques is extending our knowledge of the three-dimensional structure of mesoscale meteorological phenomena such as tornadoes, downbursts, supercells, hailstorms, and squall lines. Among these methods, the use of dynamic and kinematic equations as strong (exact) or weak (relative) constraints in a variational framework [known as three-dimensional variational data assimilation (3DVAR) or four-dimensional variational data assimilation (4DVAR)] has proven most successful (Sasaki 1970a,b). However, the underdeterminancy and strong nonlinearity of the problem, in some situations, makes difficult the identification of a unique, converged solution (Kapitza 1991).

In order to solve this problem, a nearby sounding often is used to provide background information (Sun and Crook 1998; Gao et al. 1999, 2001). However, observations from the rawinsonde network are very sparse and infrequent (launches every $12 \mathrm{~h}$ ), making it difficult to blend their information with that observed by the Weather Surveillance Radar-1988 Doppler (WSR-88D), which provides full-volume scans every 5-10 min. Reliable VAD winds could provide much needed background information for these 3DVAR or 4DVAR methods. Finally, as pointed out by Eilts and Smith (1990), VAD can provide a background wind profile for velocity dealiasing algorithms. This is especially true when the VAD method is modified based on the method of Tabary et al. (2001), as shown by Gong et al. (2003), or based on the method developed in this paper.

Despite the tremendous utility of VAD winds, the underlying method is limited by its sensitivity to instrument errors, small amplitude of radial winds, outlier observations, and migrating bird "winds" (Collins 2001). Caya and Zawadzki (1992) showed that, if the linearity assumption is violated, the winds from a VAD analysis may be erroneous. The operational assimilation of VAD wind profiles from WSR-88Ds into the National Centers for Environmental Prediction (NCEP) Eta 3D variational assimilation system began in July 1997. However, when many problems associated with VAD quality became evident, their operational usage ceased in January 1999. Since then, a quality control algorithm has been developed to deal with several sources of errors (Collins 2001).

The purpose of this paper is to study the sensitivity of VAD winds to velocity folding and other sources of errors and to explore methods for reducing them. Recently, Tabary et al. (2001) developed a method to estimate the vertical wind profile from azimuthal gradients of radar data based upon the original VAD technique. It has a potential to improve the quality of the vertical wind profile based on radar radial velocity observations. However, the method uses several subjective thresholds, and the processing of data in regions where aliased values adjoin nonaliased values is problematic. In this paper, we further modify and simplify the VAD technique to obtain vertical wind profiles using velocity gradient information. Azimuth gradients are calculated using a more objective method than in Tabary et al. (2001). Furthermore, we compare the performance of our approach with traditional VAD methods using a simulated dataset.

The paper is organized as follows: In section 2, the proposed method is described in detail, while in section 3 , it is tested using simulated data created from a single sounding from the 3 May 1999 Oklahoma City, Oklahoma, tornado outbreak. In section 4, the proposed method is applied to more than 200 volume scans of WSR-88D data collected during the 3 May 1999 Oklahoma City tornado outbreak, and the vertical wind profiles obtained are compared to those from a nearby wind profiler at Purcell, Oklahoma. A summary and concluding remarks are given in section 5 . 


\section{Methodology}

Browning and Wexler (1968) showed that if a local wind field varies almost linearly, then the horizontal velocity components can be approximated by a Taylor series expansion limited to first derivatives as follows:

$$
\begin{aligned}
& u=u_{0}+\frac{\partial u}{\partial x} x+\frac{\partial u}{\partial y} y, \\
& v=v_{0}+\frac{\partial v}{\partial x} x+\frac{\partial v}{\partial y} y,
\end{aligned}
$$

where the subscript 0 , in the first term, denotes the value at the center of the circle being scanned. The horizontal coordinates of a data point can be expressed as

$$
\begin{aligned}
& x=r \cos \theta \sin \phi, \\
& y=r \cos \theta \cos \phi,
\end{aligned}
$$

where $r=|\mathbf{x}|$ is the distance from the radar (center of circle $), \mathbf{x}=(x, y, z)$ is the position of the data point in the local three-dimensional Cartesian coordinates with the radar as the origin, $\theta$ is the elevation angle, and $\phi$ is the azimuthal angle (clockwise with respect to the $y$ coordinate pointing to north). The radial velocity, denoted $V_{r}$, is the component of the particle velocity $(u$, $v, w-w_{t}$ ) along the radar radial direction, and $w_{t}$ is terminal fall velocity of scattering targets. Radial velocity $V_{r}$ can be written as

$V_{r}=u \cos \theta \sin \phi+v \cos \theta \cos \phi+\left(w-w_{t}\right) \sin \theta$. (3)

Substituting (1) and (2) into (3) gives

$$
V_{r}=a_{0}+\sum_{n=1}^{2}\left(a_{n} \cos n \phi+b_{n} \sin n \phi\right),
$$

where the Fourier coefficients are given by

$$
\begin{aligned}
& a_{0}=\frac{1}{2} r \cos ^{2} \theta\left(\frac{\partial u}{\partial x}+\frac{\partial v}{\partial y}\right)+\left(w-w_{i}\right) \sin \theta \\
& a_{1}=v_{0} \cos \theta \\
& b_{1}=u_{0} \cos \theta \\
& a_{2}=-\frac{1}{2} r^{2} \cos ^{2} \theta\left(\frac{\partial u}{\partial x}-\frac{\partial v}{\partial y}\right) \\
& b_{2}=\frac{1}{2} r \cos ^{2} \theta\left(\frac{\partial u}{\partial y}+\frac{\partial v}{\partial x}\right) .
\end{aligned}
$$

In the traditional VAD method, the first Fourier coefficient in (4) is used directly to fit the data on the circle of a radar scan so that five parameters, $a_{0}, a_{1}, b_{1}$, $a_{2}, b_{2}$, are estimated. These parameters are used to obtain the mean wind and other kinematic information within the circle. Classic VAD requires that the radar velocity data be dealiased and pass through several quality control steps. The modified VAD technique proposed by Tabary et al. (2001) has no such requirement and in fact relies upon azimuthal gradients of radial velocity that can be obtained by taking the derivative of (4) with respect to azimuth

$$
\frac{\partial V_{r}}{\partial \phi}=\sum_{n=1}^{2} n\left(b_{n} \cos n \phi-a_{n} \sin n \phi\right) .
$$

If azimuthal gradients $\partial V_{r} / \partial \phi$ are estimated along a circle at each range, the mean wind $u_{0}, v_{0}$ easily can be obtained by least squares fitting using (10). The advantage of this methodology is that, if estimated correctly, the azimuthal wind gradients cannot be influenced by aliasing errors. Tabary et al. (2001) called this procedure the modified VAD technique. The shortcoming is that divergence information is lost because $a_{0}$ disappears from Eq. (10). However, if we wish only to obtain the vertical profile of the horizontal wind, the use of Eq. (10) is preferable in an operational context.

Estimating the gradients of radial velocity at each range is crucial to the success of the method, and Tabary et al. (2001) obtained them using a least squares fit. In the present paper, we calculate azimuthal gradients of radial velocity very simply by taking the difference of radial velocity at adjacent points in the azimuthal direction, that is,

$$
\left(\frac{\partial V_{r}}{\partial \phi}\right)_{\mathrm{ob}}^{j}=\left(\frac{V_{r}^{j+1}-V_{r}^{j}}{\phi^{j+1}-\phi^{j}}\right)_{\mathrm{ob}},
$$

where $j$ is the index of the data point in the azimuthal direction. Although azimuthal gradients obtained in this manner can be noisy, extraordinarily large gradients (by several orders of magnitude) caused either by large differences between the aliased and nonaliased data, or by large random errors, are easily be identified and flagged. They subsequently may be rejected and replaced by an average of azimuthal gradients from nearby data points. Thus, the total number of valid gradients is nearly the same as the number of original data points.

At this stage in the procedure, azimuthal gradients are free of aliasing errors. However, they still contain random noise with perhaps large amplitude. To deal with this problem, we apply a simple low-pass filter to the gradients,

$$
\left(\frac{\partial V_{r}}{\partial \phi}\right)_{\mathrm{ob}}^{k}=\sum_{j=k-N / 2}^{k+N / 2} W_{j}\left(\frac{\partial V}{\partial \phi}\right)_{\mathrm{ob}}^{j} / \sum_{j=k-N / 2}^{k-N / 2} W_{j},
$$

where $k$ is the index along a data circle in an azimuthal direction, $N$ is the total number of points to be used in the filter, and $W_{j}$ is a weighting function that has the following formulation:

$$
W_{j}=1-\frac{\operatorname{ABS}(j-k)}{N},
$$

where ABS denotes the absolute value.

In the final step, the coefficients $a_{1}, b_{1}, a_{2}$, and $b_{2}$ respectively, in Eqs. (6), (7), (8), and (9), and thus $u_{0}$, $v_{0}$ and wind deformation fields, $[(\partial u / \partial x)-(\partial v / \partial y)]$ and 
$[(\partial u / \partial y+(\partial v / \partial x)]$, are then obtained by performing a least squares fit of azimuthal gradients following application of the low-pass filter in Eq. (12).

In Tabary et al. (2001), estimating the gradient of radial velocity at each range requires about 50 radial velocity observations and the setting of several empirical threshold parameters. The total number of gradients obtained may be significantly less than the total number of data available. Thus, when the method is applied to a region in which data coverage is very sparse, it may fail. The processing of data in regions where aliased values adjoin nonaliased values is problematic.

The merit of our method is that calculation of radial velocity gradients with respect to azimuth is really a local process and does not require information regarding the Nyquist interval in which the data belong. Because only wind gradients are used, we call the technique the gradients of velocity-azimuthal display (GVAD) method.

Because our goal is to retrieve the vertical profile of the horizontal wind from Doppler radar data, only terms $a_{1}, b_{1}$ in Eq. (10) are calculated in the experiments that follow. Our results show that GVAD is rather insensitive to systematic errors caused by velocity ambiguities and to random error associated with radar instrument noise.

\section{Tests using simulated data}

\section{a. Experiment design}

To evaluate the performance of GVAD, we utilize a set of simulated single-Doppler radar data. A rawinsonde observation taken near Oklahoma City, Oklahoma, at 1800 UTC on 3 May 1999, prior to the formation of several very strong tornadic supercell storms, is used to create a volume scan of simulated WSR-88D radial velocities. The gate spacing in the radial direction (range resolution) is $250 \mathrm{~m}$ with a maximum range of $230 \mathrm{~km}$ and an azimuth resolution of $1^{\circ}$. A full azimuthal sweep at several elevation angles is made, similar to the 14 elevation angles used in the WSR-88D severe weather antenna scanning strategy during precipitation. The number of grid points in the radial and azimuthal directions is 920 and 360, respectively, for each tilt. Figure 1a shows one tilt of these simulated radial velocities at an elevation of approximately $5^{\circ}$, which is close to one of the real elevation angles used by the WSR-88D.

The simulated aliased radial velocity is given by

$$
V_{\mathrm{rm}}=V_{r}-2 n V_{a},
$$

where $V_{a}$ is the maximum unambiguous velocity, or Nyquist velocity of the radar; $V_{r}$ is the true radial velocity created from the sounding data; and the integer $n$ is termed the Nyquist folding number and represents the number of Nyquist intervals in which the true velocity deviates from the measured value. Note that $n$ can be positive or negative.
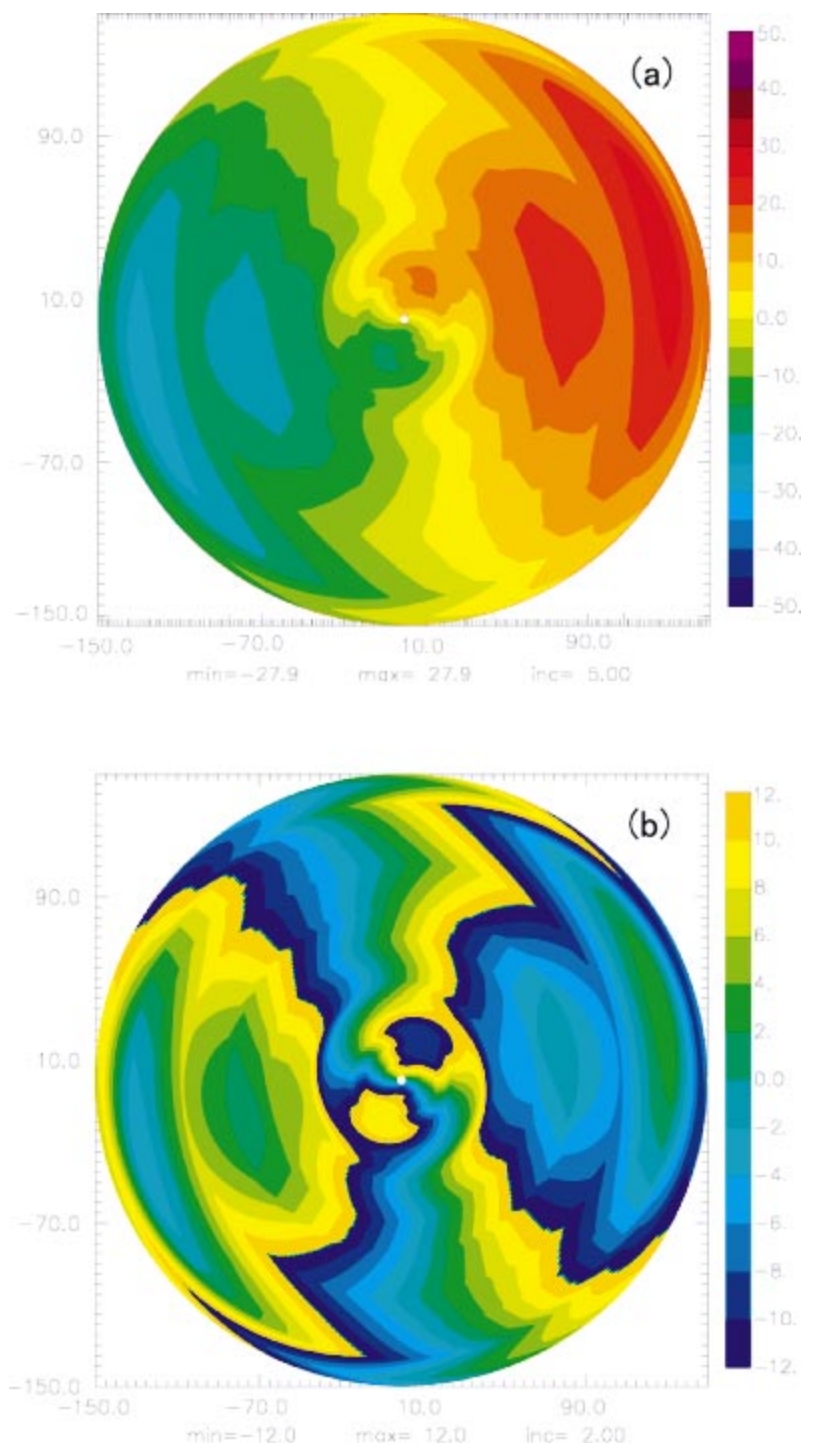

FIG. 1. (a) Simulated radial velocity field derived from 1800 UTC 3 May 1999 sounding. (b) The aliased radial velocity field assuming a Nyquist velocity of $12 \mathrm{~m} \mathrm{~s}^{-1}$ and no random errors in the data (no instrumental noises or other errors).

In the following experiments, we create two instances of $V_{\mathrm{rm}}$ by setting the unambiguous velocity, $V_{a}$, to 12 and $23 \mathrm{~m} \mathrm{~s}^{-1}$ in order to represent situations in which the WSR-88D operates in clear-air and precipitation modes, respectively. Figure $1 \mathrm{~b}$ is obtained using (13) when the unambiguous velocity, $V_{a}$, is $12 \mathrm{~m} \mathrm{~s}^{-1}$. Almost half of the radial velocity data are contaminated by aliasing.

In order to make our simulated experiments as close as possible to the use of real WSR-88D data, random errors are added to $V_{r}$ or $V_{\mathrm{rm}}$. The error formulation for $V_{r}$ is

$$
V_{r}^{\prime}=V_{r}+\alpha \varepsilon(0,1)
$$

where $\alpha$ is the error amplitude, and $\varepsilon$ is a random num- 
ber having a normal Gaussian distribution with zero mean and unity standard deviation. Random errors with different amplitudes are added to the radial velocities in most of the following experiments. An error amplitude $\alpha$ of $1 \mathrm{~m} \mathrm{~s}^{-1}$ corresponds to the standard accuracy of observed radial winds for the WSR-88D.

The simulated WSR-88D radial velocity data are used to retrieve a single vertical wind profile using the traditional VAD technique as well as GVAD for comparison against the reference "truth." To measure the accuracy of GVAD and VAD, we calculate the rms error and relative rms error (RRE) of the wind direction and speed:

$$
\begin{gathered}
\text { rms_P }=\sqrt{\frac{\sum_{i=1}^{N}\left(p-p_{\text {ref }}\right)_{i}^{2}}{N}}, \\
\text { RRE_P }=\sqrt{\frac{\sum_{i=1}^{N}\left(p-p_{\text {ref }}\right)_{i}^{2}}{\sum_{i=1}^{N}\left(p_{\text {ref }}\right)_{i}^{2}}} .
\end{gathered}
$$

Here $p$ can either be the calculated gradient, the wind direction, or the wind speed, and $N$ represents either the total number of gradients of radial velocity in radar coverage, the number of retrieved wind directions, or the number of retrieved wind speeds. The subscript ref refers to the "truth" for gradients or the wind profile. In addition, the correlation coefficients (CCs) of wind direction and speed between the retrieved and "true" profiles are also calculated for each experiment.

\section{b. Accuracy of calculated azimuthal gradients}

The reliability of GVAD depends upon the accuracy of the computed azimuthal gradients of radial velocity, $\partial V_{r} / \partial \phi$. The first experiment, shown in the first row of Table 1 , is a case in which the simulated radial velocities $V_{r}$ contain neither random errors $(\alpha=0.0)$ nor aliasing errors (Fig. 1a). We find that the azimuth gradients calculated using Eq. (11) are almost identical to the true ones derived from Eq. (10), with an rms equal to only $0.107 \mathrm{~m} \mathrm{~s}^{-1}$ radian $^{-1}$. The second experiment in Table 1 is identical to the first, though with aliasing errors caused by a Nyquist velocity of $12 \mathrm{~m} \mathrm{~s}^{-1}$ (Fig. 1b). In this case, the quality of the calculated gradients is nearly the same as the case without aliasing errors (i.e., the rms errors are almost same).

Figure 2a shows azimuth gradients computed from perfect radial velocity data (see Fig. 1a), while Fig. 2b shows the same from data containing large aliasing errors (see Fig. 1b). The two figures are nearly identical, except for the presence of discontinuities in Fig. 2b where aliased values of radial velocity adjoin nonaliased values, and thus where the calculated gradients are several orders of magnitude larger than those of their neighbors. The spurious gradients are easily identified and replaced by an average of gradients from nearby points.

If the error amplitude $\alpha$ in Eq. (14) is set to $1 \mathrm{~m} \mathrm{~s}^{-1}$ that is, the standard accuracy of observed radial velocities - the calculated gradients using only Eq. (11) contain large random errors. Relative rms errors for both cases (with and without aliasing errors) exceed 600\%, and the correlation coefficient between the calculated and true gradients falls to 0.149 . When we increase $\alpha$ to $3 \mathrm{~m} \mathrm{~s}^{-1}$, the relative errors exceed $2000 \%$ and the correlation coefficient reduces to 0.04 . This indicates that the calculated gradients using only Eq. (11) are very sensitive to random noise in the "observed" radial velocity data regardless of whether the radial velocity is aliased.

Figure $3 \mathrm{a}$ shows that no coherent structures are present in the gradient field when the simulated radial velocities contain both random and systematic (aliasing) errors. However, it is easy to separate these errors because the discontinuities are caused by gradients that are orders of magnitude larger than normal. While large noise masks the coherent structures in Fig. 3a, it can be removed using the low-pass filter (12).

In the second part of Table 1, we perform experiments in which the low-pass filter (12) is applied to gradients (see Fig. 3a) after aliasing-gradient discontinuities have been removed. The quality of the computed gradients is greatly improved because the rms and relative rms errors are significantly reduced (Table 2). Figure $3 b$ shows results obtained following the application to gradients in Fig. 3a of the low-pass filter. The resultant gradients clearly are much closer to the true ones shown in Fig. 2a.

This series of experiments demonstrates that random errors are amplified in the calculated azimuthal wind gra-

\begin{tabular}{|c|c|c|c|c|c|c|c|}
\hline \multirow{2}{*}{\multicolumn{2}{|c|}{$\Delta V_{r} / \Delta \theta$}} & \multicolumn{3}{|c|}{ Without aliasing $\left(V_{\mathrm{a}}=0\right)$} & \multicolumn{3}{|c|}{ With aliasing $\left(\mathrm{V}_{a}=12 \mathrm{~m} \mathrm{~s}^{-1}\right)$} \\
\hline & & Rms & RRE (\%) & $\mathrm{CC}$ & Rms & RRE (\%) & $\mathrm{CC}$ \\
\hline \multirow[t]{3}{*}{ No filter } & $\alpha=0.0$ & 0.107 & 0.871 & 1.000 & 0.106 & 0.896 & 1.000 \\
\hline & $\alpha=1.0$ & 81.16 & 662.5 & 0.149 & 81.17 & 662.4 & 0.149 \\
\hline & $\alpha=3.0$ & 2435 & 1987.3 & 0.050 & 246.4 & 2014 & 0.043 \\
\hline \multirow[t]{3}{*}{ With filter } & $\alpha=0.0$ & 0.483 & 3.943 & 1.000 & 0.489 & 3.989 & 1.000 \\
\hline & $\alpha=1.0$ & 1.437 & 11.73 & 0.993 & 2.507 & 20.46 & 0.979 \\
\hline & $\alpha=3.0$ & 4.105 & 33.51 & 0.945 & 8.252 & 67.367 & 0.789 \\
\hline
\end{tabular}

TABLE 1. Sensitivity of calculated radial gradients to the errors in the simulated radial velocities. 

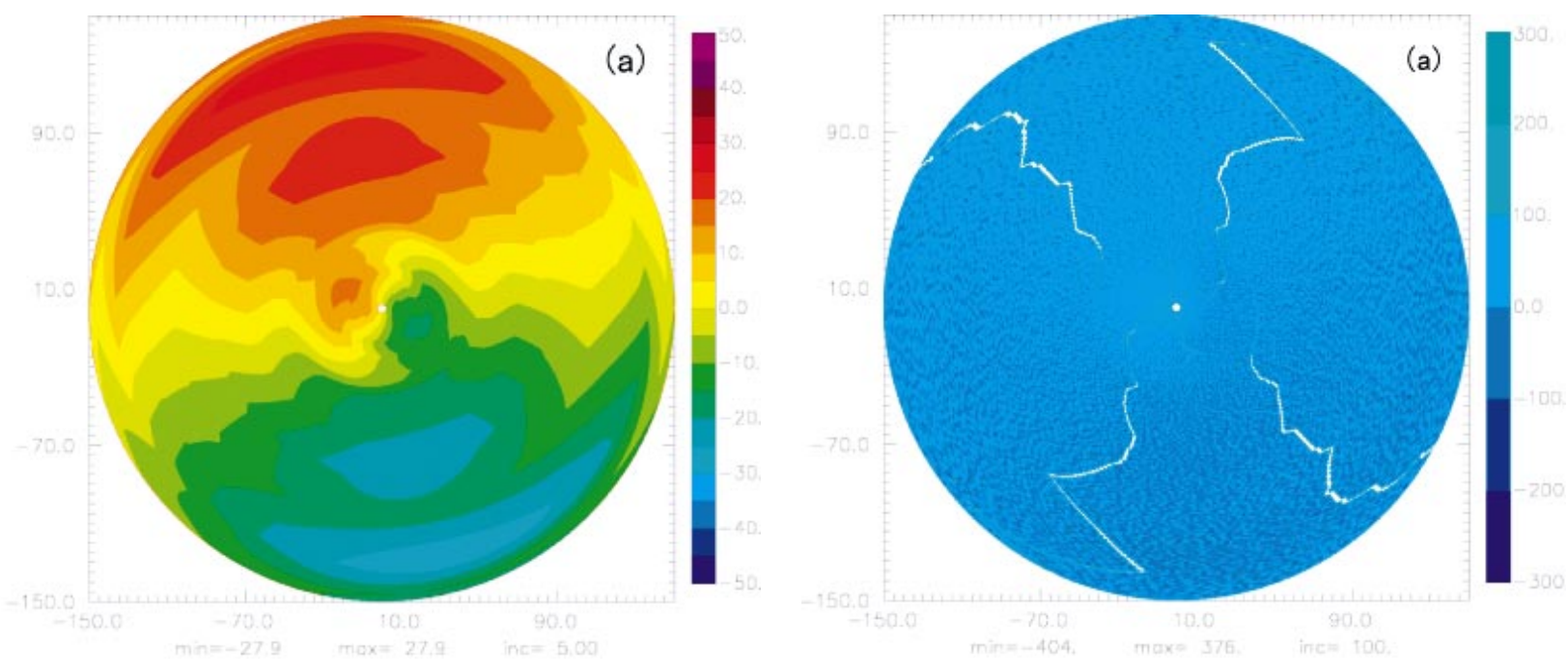

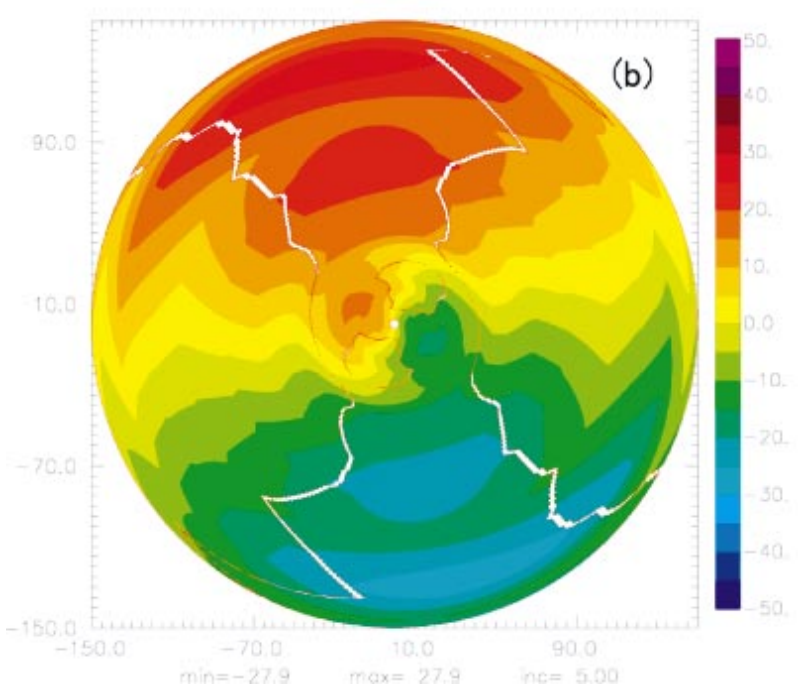

FIG. 2. The gradients of radial velocity with respect to azimuth angle calculated from difference scheme (11): (a) using the radial velocity data shown in Fig. 1a, and (b) using the radial velocity data shown in Fig. $1 b$.

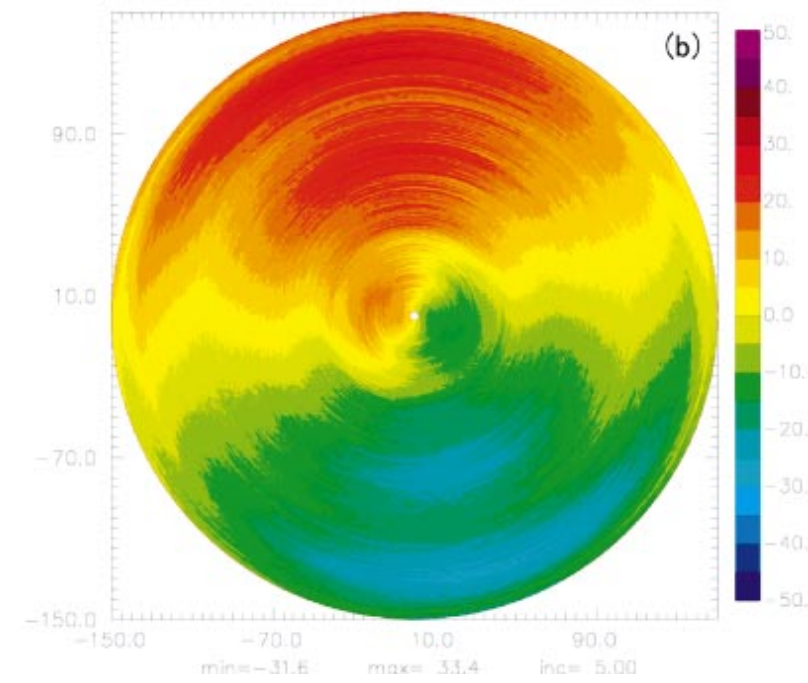

FIG. 3. Same as Fig. 2, but using a different radial velocity dataset. (a) The data contain random errors with amplitude $1 \mathrm{~m} \mathrm{~s}^{-1}$. (b) Applying a low-pass filter to the calculated azimuth gradients shown by (a), but after the discontinuities are removed.

TABLE 2. Sensitivity of VAD and GVAD results to random errors in radial velocities without aliasing errors.

\begin{tabular}{|c|c|c|c|c|c|c|c|}
\hline \multirow{2}{*}{\multicolumn{2}{|c|}{ Errors and coefficients }} & \multicolumn{3}{|c|}{ Wind direction $\left({ }^{\circ}\right)$} & \multicolumn{3}{|c|}{ Wind speed $\left(\mathrm{m} \mathrm{s}^{-1}\right)$} \\
\hline & & Rms & RRE (\%) & $\mathrm{CC}$ & Rms & $\operatorname{RRE}(\%)$ & $\mathrm{CC}$ \\
\hline \multirow[t]{2}{*}{$\alpha=0.0$} & VAD & $1.457 \times 10^{-5}$ & $6.068 \times 10^{-6}$ & 1.000 & $4.753 \times 10^{-6}$ & $2.735 \times 10^{-5}$ & 1.000 \\
\hline & GVAD & 0.500 & 0.208 & 1.000 & 0.668 & 3.846 & 1.000 \\
\hline \multirow[t]{2}{*}{$\alpha=1.0$} & VAD & 0.536 & 0.223 & 1.000 & $9.413 \times 10^{-2}$ & 0.542 & 1.000 \\
\hline & GVAD & 3.378 & 1.407 & 0.992 & 0.934 & 5.377 & 0.994 \\
\hline \multirow[t]{2}{*}{$\alpha=3.0$} & VAD & 1.628 & 0.678 & 0.998 & 0.282 & 1.625 & 0.999 \\
\hline & GVAD & 10.88 & 4.53 & 0.930 & 2.054 & 11.82 & 0.947 \\
\hline
\end{tabular}



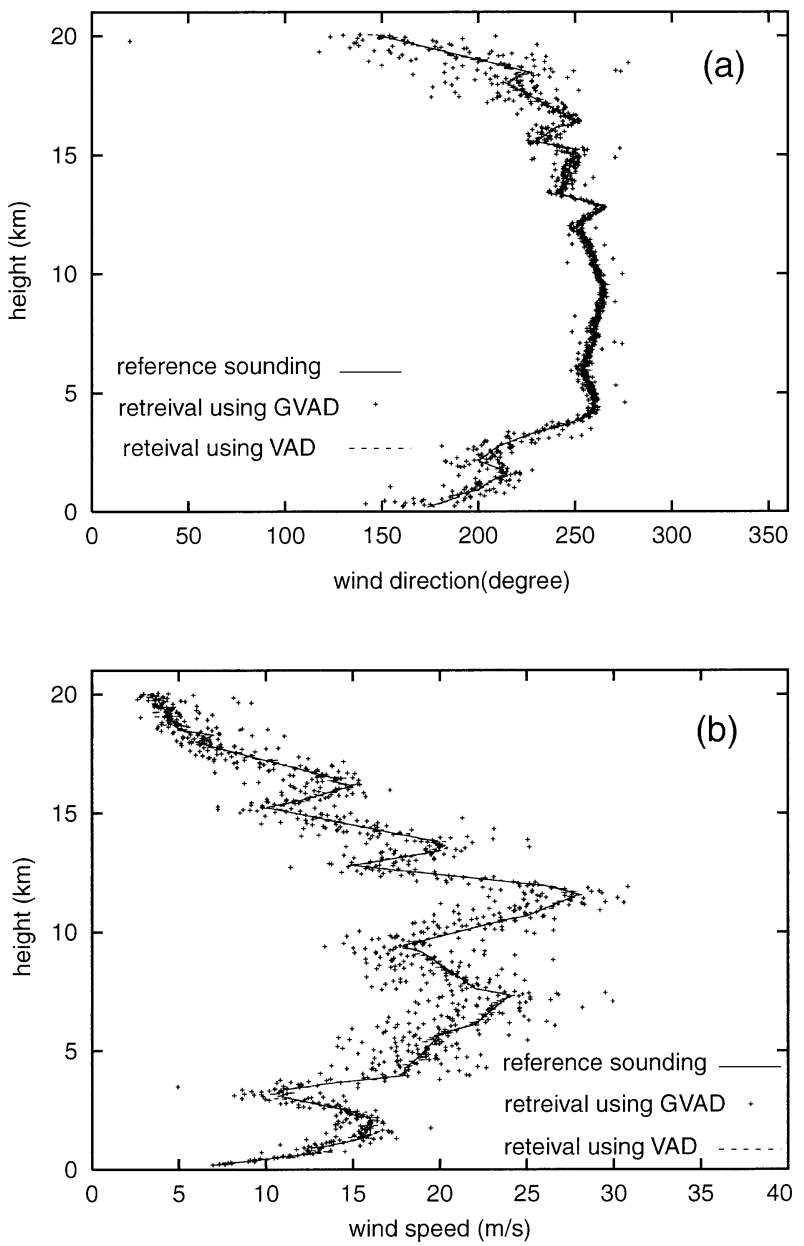

FIG. 4. The plotting of true and retrieved wind profile versus height without alias errors but with random errors when $\alpha$ equals $3.0 \mathrm{~m} \mathrm{~s}^{-1}$. (a) The profile of wind direction, and (b) the profile of wind speed.

dient field due to finite differencing. However, because large systematic errors associated with velocity aliasing already have been removed, a low-pass filter (12) can be applied to effectively remove random errors and further improve the quality of the computed gradients.

\section{c. Results of retrieved GVAD and VAD winds}

\section{1) SEnsitivity to RANDOM ERRORS Without} ALIASING CONTAMINATION

In this section (see Table 2) we present results of GVAD and VAD using simulated radial velocity data that contains no aliasing errors, only random errors with varying amplitudes. The quality of the retrieval can be ascertained by examining rms errors and correlation coefficients for wind direction and speed and by comparing the retrieved fields with the "truth," that is, the sounding at 1800 UTC on 3 May 1999.

In the first experiment, the input data, or the simulated radial velocities, are perfect and contain no velocity folding or random errors. Table 2 shows that the rms and relative rms errors for wind speed and direction are very small for both VAD and GVAD. However, VAD is slightly superior because GVAD, which uses gradients of radial velocities calculated from (11), introduces errors associated with the finite-differencing process.

In the second experiment in Table 2, the error amplitude $\alpha$ in Eq. (14) is set to $1 \mathrm{~m} \mathrm{~s}^{-1}$. The wind profile retrieved using GVAD is comparable to that obtained using VAD. Figure 4 compares both retrieved profiles with the "truth," and the agreement is reasonably good. We thus conclude that wind profiles calculated using both VAD and GVAD are fairly insensitive to random errors in radial velocity data, and that VAD is slightly better than GVAD in the absence of aliasing errors.

\section{2) Sensitivity to Aliasing CONTAmination}

The second series of experiments is designed to test the sensitivity of both VAD and GVAD to systematic errors (aliasing errors) in the presence of random errors (amplitude $\alpha=1.0 \mathrm{~m} \mathrm{~s}^{-1}$ ) in the simulated radial velocities. In the first experiment in Table 3, we set the Nyquist velocity to $23 \mathrm{~m} \mathrm{~s}^{-1}$, which is close to the limit of the WSR-88D operating in precipitation mode. About $3 \%$ of the simulated radial velocities are contaminated by aliasing errors. Table 3 indicates that GVAD performs much better than VAD under these circumstances. The rms wind direction error for the retrieved wind profile using GVAD is only $3.45^{\circ}$, and the speed error is only $0.94 \mathrm{~m} \mathrm{~s}^{-1}$. In the case of VAD, the errors are $43.04^{\circ}$ and $7.01 \mathrm{~m} \mathrm{~s}^{-1}$, respectively. The correlation coefficients for both methods also indicate that GVAD is superior to VAD. In fact, Fig. 5 shows that the retrieved wind profile using GVAD is reasonably close to the true one, while that obtained from VAD exhibits notable errors at several levels.

In the second experiment, the Nyquist velocity is set to $12 \mathrm{~m} \mathrm{~s}^{-1}$ (Table 3 ) so that $39 \%$ of the simulated radial velocity field is contaminated by aliasing error. In this case, VAD fails completely (with large rms, relative rms

TABLE 3. Sensitivity of VAD and GVAD results to random errors in radial velocities with aliasing errors $(\alpha=1.0)$.

\begin{tabular}{|c|c|c|c|c|c|c|c|}
\hline \multirow{2}{*}{$\begin{array}{l}\text { Nyquist } \\
\text { velocity }\end{array}$} & \multirow[b]{2}{*}{ Method } & \multicolumn{3}{|c|}{ Wind direction $\left(^{\circ}\right)$} & \multicolumn{3}{|c|}{ Wind speed $\left(\mathrm{m} \mathrm{s}^{-1}\right)$} \\
\hline & & Rms & RRE (\%) & $\mathrm{CC}$ & Rms & RRE (\%) & $\mathrm{CC}$ \\
\hline \multirow[t]{2}{*}{$23 \mathrm{~m} \mathrm{~s}^{-1}$} & VAD & 43.05 & 17.93 & 0.437 & 7.017 & 40.38 & 0.413 \\
\hline & GVAD & 3.454 & 1.439 & 0.992 & 0.941 & 5.415 & .994 \\
\hline \multirow[t]{2}{*}{$12 \mathrm{~m} \mathrm{~s}^{-1}$} & VAD & 148.1 & 61.71 & -0.34 & 14.07 & 80.99 & -0.44 \\
\hline & GVAD & 5.882 & 2.450 & 0.977 & 1.480 & 8.519 & 0.978 \\
\hline
\end{tabular}



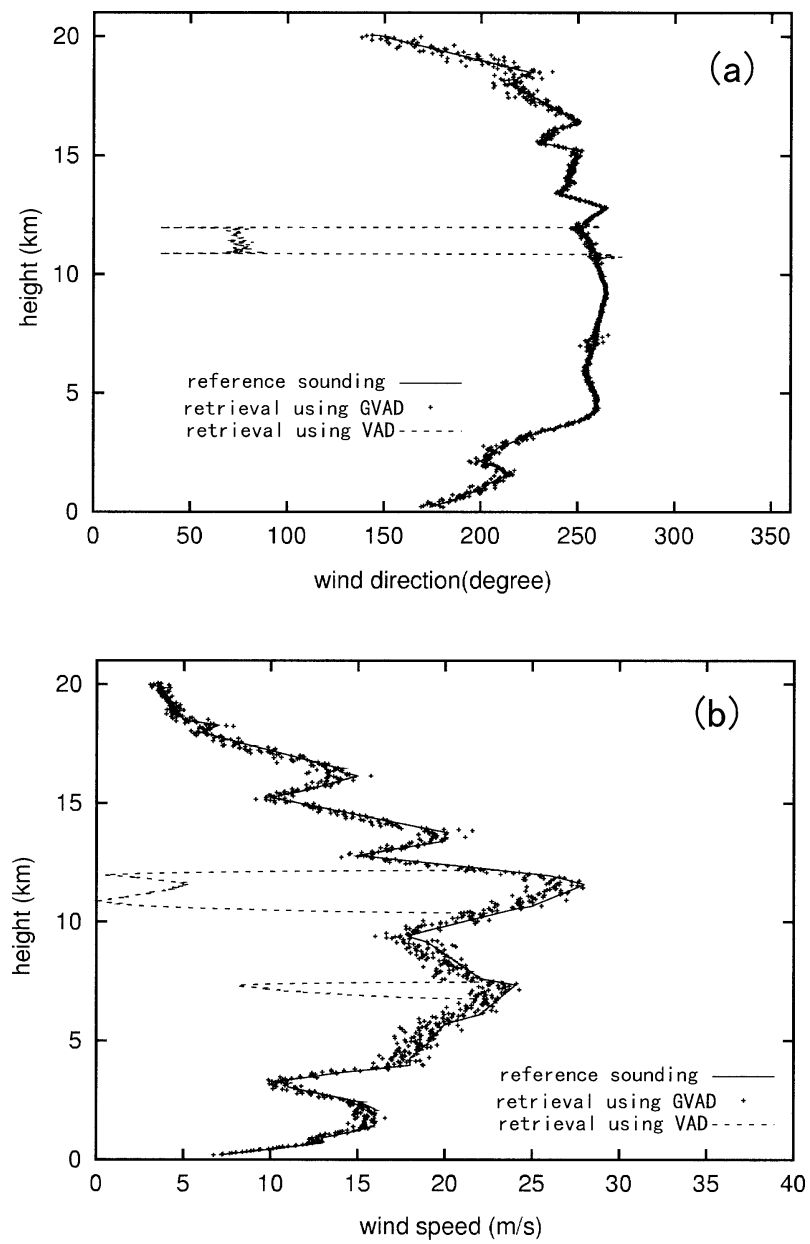

Fig. 5. As in Fig. 4, but for the case when the data contain minor alias errors $(3 \%)$ when Nyqiust velocity is $23 \mathrm{~m} \mathrm{~s}^{-1}$, and random errors when $\alpha$ equals $1.0 \mathrm{~m} \mathrm{~s}^{-1}$.

errors, and negative correlation coefficients), while GVAD still provides reasonable wind profile information. These results suggest that traditional VAD may fail even when only a small percentage of data are contaminated by aliasing errors; in contrast, GVAD is quite robust.

\section{3) LIMITED RADAR COVERAGE EXPERIMENTS}

In the previous two subsections, we examined the sensitivity of VAD and GVAD to random and systematic errors. In this section, we focus on the sensitivity of GVAD to data coverage. To maximize the realism of the experiments, we set the random error amplitude, $\alpha$ in Eq. (14), to $1 \mathrm{~m} \mathrm{~s}^{-1}$. The Nyquist velocity is set to $12 \mathrm{~m} \mathrm{~s}^{-1}$ so that about $39 \%$ of the data are contaminated by aliasing errors.

The results are presented in Table 4, and for convenience in comparison, the results using full data coverage from the last experiment also are listed. In the first experiment, only half of the full-volume scan (from $0^{\circ}$ to $180^{\circ}$ in azimuth direction) contains radial velocity data. As shown in the second row of Table 4, the correlation coefficients for both wind direction and speed are still larger than 0.9 , and relative rms errors are much less than $10 \%$.

In the second experiment (last row of Table 4), the data coverage is reduced to one-tenth of a full-volume scan (from $1^{\circ}$ to $10^{\circ}$ in azimuth). The quality of the retrieved wind profile is greatly reduced, and large bias errors clearly are present. Figure 6 shows the associated retrieved wind profile. Even when smoothed in the vertical, it still contains large bias errors. This result suggests a limitation when applying GVAD in data-sparse regions.

\section{Tests using real WSR-88D data}

To further test the performance of GVAD, we apply it to the 3-4 May 1999 tornado outbreak in the southern Great Plains. On that day, nine supercell thunderstorms occurred in Oklahoma, and violent tornadoes produced significant damage. Doppler radar data collected over a period of $31 \mathrm{~h}$ (more than 200 volume scans from 0013 UTC on 3 May to 0704 UTC on 4 May) from the KTLX WSR-88D are used. This is a very challenging case because radial velocity measurements were severely contaminated by large aliasing errors, especially in the vicinity of observed mesocyclones and tonadoes. During this same time period, vertical wind profile observations were collected from the Purcell, Oklahoma, wind profiler ( $25 \mathrm{~km}$ south of the KTLX radar) every hour and are used to verify the performance of GVAD.

Figure 7 shows the scatterplots of wind direction and speed as derived from the KTLX radar using GVAD and as measured by the Purcell wind profiler. The rms errors for wind direction and speed are $11.80^{\circ}$ and 3.97 $\mathrm{m} \mathrm{s}^{-1}$, respectively, and the correlation coefficients are 0.81 and 0.77 , respectively. These statistics are quite good for such a challenging case. To further quantify the results, Fig. 8 shows the same information at 0000

TABLE 4. Sensitivity of GVAD results to data coverage (Nyquist velocity is $12 \mathrm{~m} \mathrm{~s}^{-1}$, and $\alpha=1.0$ ).

\begin{tabular}{|c|c|c|c|c|c|c|c|}
\hline & & \multicolumn{3}{|c|}{ Wind direction $\left(^{\circ}\right)$} & \multicolumn{3}{|c|}{ Wind speed $\left(\mathrm{m} \mathrm{s}^{-1}\right)$} \\
\hline \multicolumn{2}{|c|}{$\%$ of data coverage } & Rms & RRE (\%) & $\mathrm{CC}$ & Rms & $\operatorname{RRE}(\%)$ & $\mathrm{CC}$ \\
\hline 100 & GVAD & 5.882 & 2.450 & 0.977 & 1.480 & 8.519 & 0.978 \\
\hline 50 & GVAD & 10.23 & 4.261 & 0.959 & 5.000 & 28.77 & 0.941 \\
\hline 10 & GVAD & 65.08 & 27.11 & 0.545 & 6.120 & 35.22 & 0.775 \\
\hline
\end{tabular}



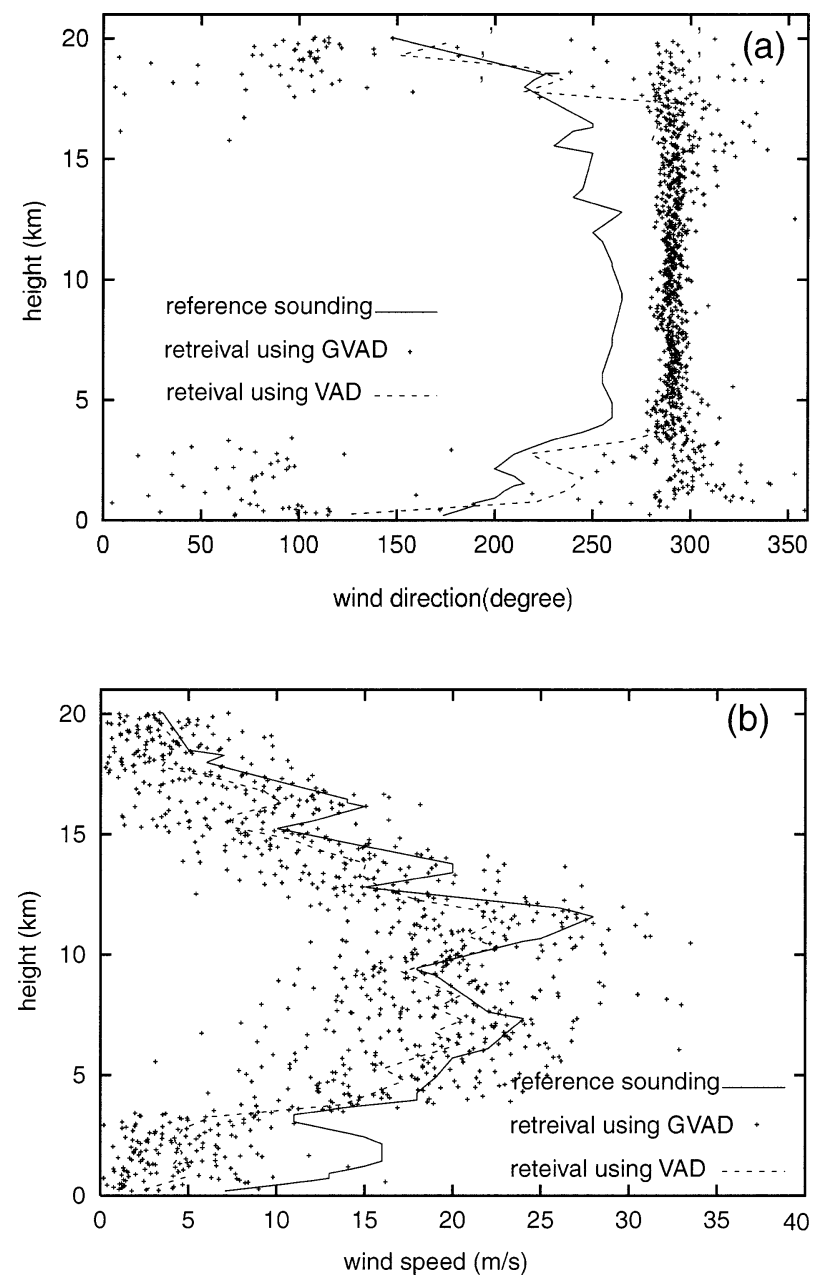

Fig. 6. As in Fig. 4, but for the case when the data contain large alias errors (39\%) when Nyqiust velocity is $12 \mathrm{~m} \mathrm{~s}^{-1}$, random errors when $\alpha$ equals $1.0 \mathrm{~m} \mathrm{~s}^{-1}$, and data coverage is only one-tenth of the total of each tilt or PPI.

UTC on 4 May 1999 when the radar was operating in precipitation mode. In general, the two profiles agree, with a relative difference less than $10 \%$. This is very satisfactory given that F4-F5-scale tornadoes were occurring at that time.

Figure 9 shows another example, at an earlier time, when the KTLX radar was operating in clear-air mode. Because the weather signal was very weak at high elevation angles, the vertical wind profile is confined to the region below 2-km altitude. Nevertheless, the profile retrieved using GVAD still agrees quite well with that obtained from the Purcell wind profiler. It is worth noting that the first level of usable wind profiler data typically is a few hundred meters above ground level; in contrast, GVAD winds can be obtained at altitudes as low as $50 \mathrm{~m}$, even when the radar is operating in clearair mode.
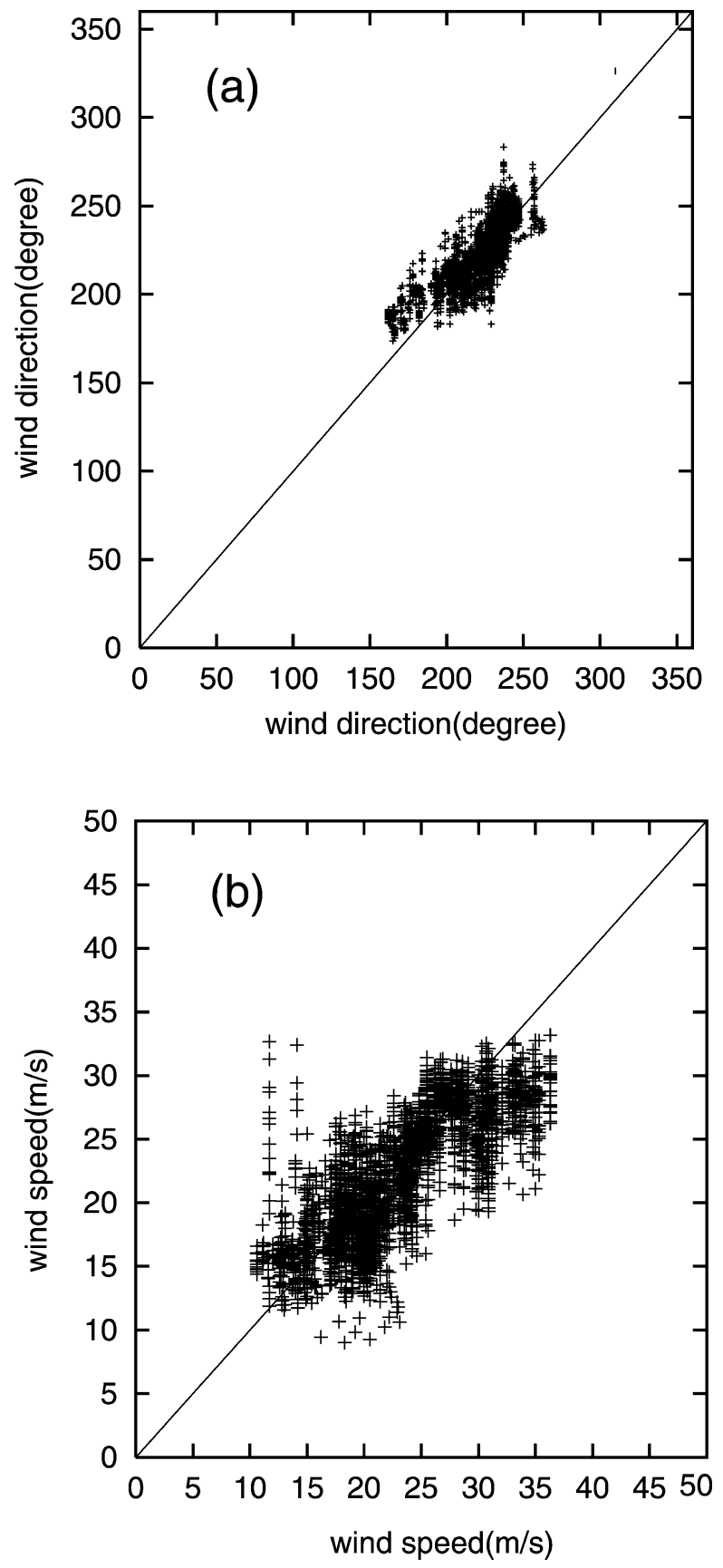

FIG. 7. Scatterplot of (a) wind direction (b) wind speed. The abscissa is for wind profiler, and the ordinate is for GVAD wind profile.

\section{Summary and concluding remarks}

We compare in this paper the performance of VAD and GVAD using simulated Doppler radar radial wind data. When these data are contaminated by large random errors, but not by aliasing errors, both VAD and GVAD perform almost equally well. However, when the data contain aliasing errors, the results from VAD are very sensitive and thus cannot be used reliably. In contrast, GVAD performs reliably. We also applied GVAD to a 

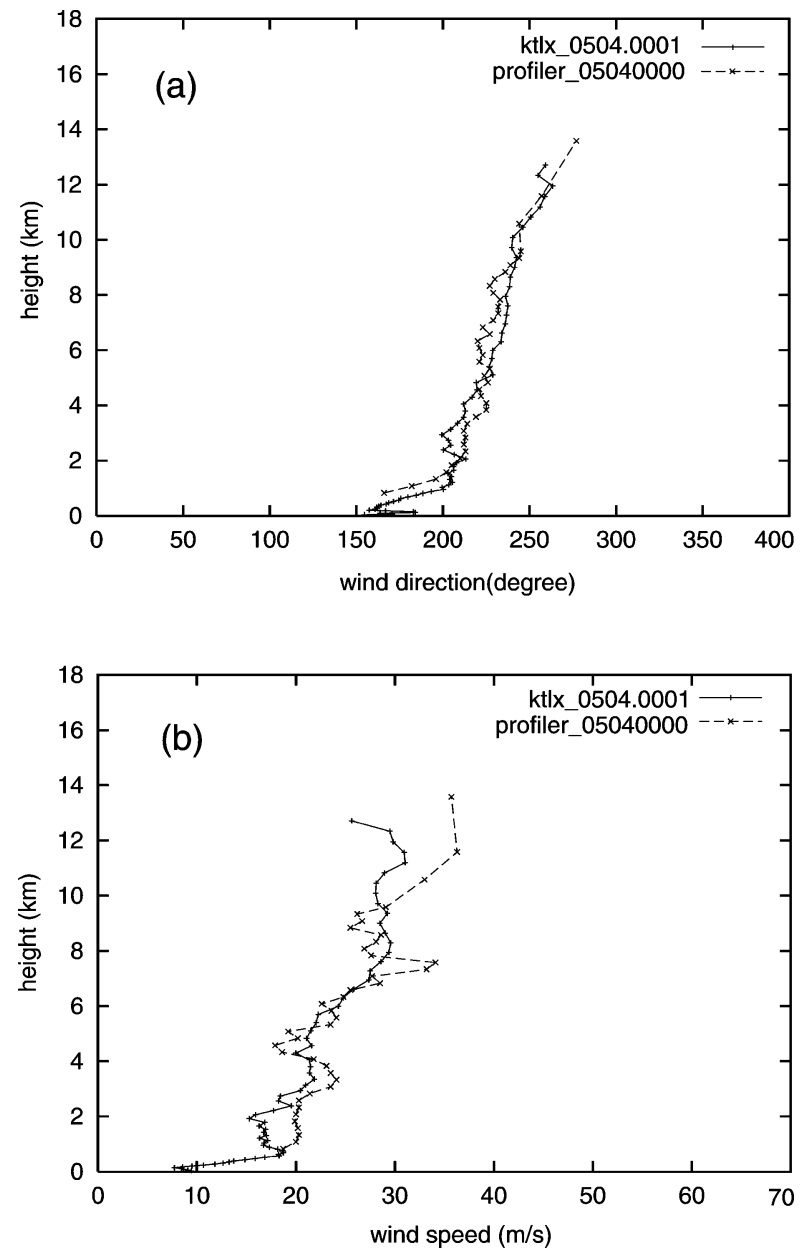

FIG. 8. Comparison of wind profile for 0000 UTC 4 May 1999 from wind profiler in Purcell, OK, and retrieved from KTLX radar using the GVAD method: (a) wind direction, and (b) wind speed.

real storm case and found good agreement between the retrieved wind profiles and those obtained from a nearby wind profiling radar.

The original VAD method, and its subsequent variants, can only be used when radar data are dealiased. If dealiasing fails or is of poor quality, the resulting VAD winds will contain significant error. Even if dealiasing is successful, it often is computationally time consuming. In this paper, we build upon the work of Tabary et al. (2001) and propose a new method that obtains directly the vertical profile of horizontal wind from WSR-88D radial velocity data. The profiles are shown to be reliable and suitable for use in large-scale data assimilation. One drawback is that wind divergence cannot be derived simultaneously. However, GVAD is extremely computationally efficient, making it especially suitable for real-time operation.

Results from GVAD also can be used as a first guess for dealiasing the radial velocity data for subsequent use in VAD, allowing one to obtain more detailed kinematic properties, such as divergence and vorticity, if neces-
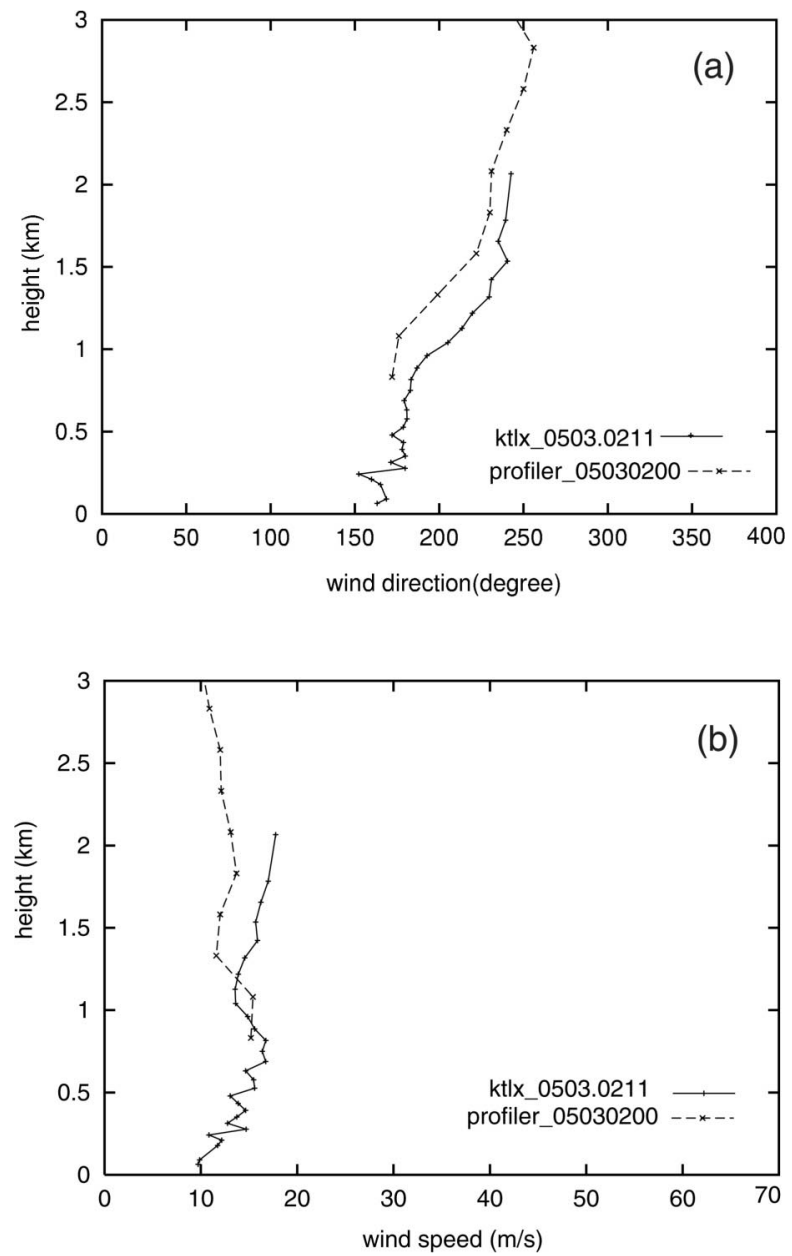

FIG. 9. Same as Fig. 8, but for 0200 UTC 3 May 1999 when the radar operated in clear-air mode.

sary. However, a very reliable dealiasing algorithm is needed. As indicated in this paper, even if only $3 \%$ of a full-volume scan of radial wind data is contaminated by aliasing errors, relative rms errors in the mean wind profile retrieved by VAD can reach $50 \%$. Thus, velocity aliasing can be a major source of error for wind profiles obtained from VAD algorithms. A robust dealiasing algorithm has been developed by Gong et al. (2003) recently.

Wind profiles obtained from GVAD also can be used to provide a background field for 3D wind retrieval techniques that utilize single- or multiple-Doppler radar data. We believe that GVAD has the potential to replace VAD in operational large-scale data assimilation and forecasting. Further, GVAD also can be easily extended to volume velocity processing, and experiments now are underway to evaluate such capability.

Acknowledgments. This research was supported by the National Science Foundation under Grants ATM 0129892, 03-31756,ATM 99-81130, and EEC03-13747, and by the Federal Aviation Administration under Grant 
NA17RJ1227-01. The authors benefited from many discussions with Drs. Alan Shapiro, Pengfei Zhang, Keith Brewster, Shun Liu, Soen-Yong Lee, and Mrs. Janelle Janish.

\section{REFERENCES}

Boccippio, D. J., 1995: A diagnostic analysis of the VVP singleDoppler retrieval technique. J. Atmos. Oceanic Technol., 12, 230-248.

Browning, K. A., and R. Wexler, 1968: The determination of kinematic properties of a wind field using Doppler radar J. Appl. Meteor., 7, 105-113.

Caya, D., and I. Zawadzki, 1992: VAD analysis of nonlinear wind fields. J. Atmos. Oceanic Technol., 9, 575-587.

Collins, W. G., 2001: The quality control of velocity azimuth display (VAD) winds at the National Centers for Environmental Prediction. Preprints, 11th Symp. on Meteorological Observations and Instrumentation, Albuquerque, NM, Amer. Meteor. Soc., 317-320.

Eilts, M. D., and S. D. Smith, 1990: Efficient dealiasing of Doppler velocities using local environment constraints. J. Atmos. Oceanic Technol., 7, 118-128.

Gao, J., M. Xue, A. Shapiro, and K. K. Droegemeier, 1999: A variational analysis for the retrieval of three-dimensional mesoscale wind fields from two Doppler radars. Mon. Wea. Rev., 127, 2128-2142.

,,,--- Q. Xu, and K. K. Droegemeier, 2001: Three dimensional simple adjoint velocity retrievals from single-Doppler radar. J. Atmos. Oceanic Technol., 18, 26-38.

Gong, J., L. Wang, and Q. Xu, 2003: A three-step dealiasing method for Doppler velocity data quality control. J. Atmos. Oceanic Technol., 20, 1738-1748.

Kapitza, H., 1991: Numerical experiments with the adjoint of a nonhydrostatic mesoscale model. Mon. Wea. Rev., 119, 2993-3011.

Koscielny, A. J., R. J. Doviak, and R. Rabin, 1982: Statistical considerations in the estimation of divergence from single-Doppler radar and application to prestorm boundary-layer observations. J. Appl. Meteor., 21, 197-210.

Lhermitte, R. M., and D. Atlas, 1961: Precipitation motion by pulse Doppler radar. Proc. Ninth Weather Radar Conf., Boston, MA Amer. Meteor. Soc., 218-223.

Matejka, T. J., 1993: Concurrent extended vertical velocity azimuth display (CEVAD). Preprints, 26th Int. Conf. on Radar Meteorology, Norman, OK, Amer. Meteor. Soc., 463-465.

_ , and R. C. Srivastava, 1991: An improved version of the extended velocity-azimuth display analysis of single Doppler radar data. J. Atmos. Oceanic Technol., 8, 453-466.

Michelson, S. A., and N. L. Seaman, 2000: Assimilation of NEXRAD-VAD winds in summertime meteorological simulations over the northeastern United States. J. Appl. Meteor., 39, 367383.
Qiu, C.-J., and Q. Xu, 1992: A simple adjoint method of wind analysis for single-Doppler data. J. Atmos. Oceanic Technol., 9, 588598.

Sasaki, Y., 1970a: Numerical variational analysis with weak constraint and application to surface analysis of severe storm gust. Mon. Wea. Rev., 98, 875-910.

__ 1970b: Some basic formalisms in numerical variational analysis. Mon. Wea. Rev., 98, 875-883.

Serafin, R. J., and J. W. Wilson, 2000: Operational weather radar in the United States: Progress and opportunity. Bull. Amer. Meteor. Soc., 81, 501-518.

Shapiro, A., S. Ellis, and J. Shaw, 1995: Single-Doppler velocity retrievals with Phoenix II data: Clear air and microburst retrievals in the planetary boundary layer. J. Atmos. Sci., 52, 12651287.

__ P. Robinson, J. Wurman, and J. Gao, 2003: Single-Doppler velocity retrieval with rapid-scan radar data. J. Atmos. Oceanic Technol., 20, 1758-1775.

Srivastava, R. C., T. J. Matejka, and T. J. Lorello, 1986: Doppler radar study of the trailing anvil region associated with a squall line. J. Atmos. Sci., 43, 356-377.

Sun, J., and N. A. Crook, 1997: Dynamical and microphysical retrieval from Doppler radar observations using a cloud model and its adjoint. Part I: Model development and simulated data experiments. J. Atmos. Sci., 54, 1642-1661.

__ and _ _ 1998: Dynamical and microphysical retrieval from Doppler radar observations using a cloud model and its adjoint. Part II: Retrieval experiments of an observed Florida convective storm. J. Atmos. Sci., 55, 1642-1661.

_, D. W. Flicker, and D. K. Lilly, 1991: Recovery of three-dimensional wind and temperature fields from simulated Doppler radar data. J. Atmos. Sci., 48, 876-890.

Tabary, P., G. Scialom, and U. Germann, 2001: Real-time retrieval of the wind from aliased velocities measured by Doppler radars. J. Atmos. Oceanic Technol., 18, 875-882.

Waldteufel, P., and H. Corbin, 1979: On the analysis of single Doppler radar data. J. Appl. Meteor., 18, 532-542.

Weygandt, S., A. Shapiro, and K. Droegemeier, 1995: Adaptation of a single-Doppler retrieval for use on a deep-convection storm. Preprints, 27th Conf. on Radar Meteorology, Vail, CO, Amer. Meteor. Soc., 264-266.

$-\ldots$, and $-\ldots$, 2002: Retrieval of model initial fields from single-Doppler observations of a supercell thunderstorm. Part I: Single-Doppler velocity retrieval. Mon. Wea. Rev., 130, 433453.

Xu, Q., and C. J. Qiu, 1994: Simple adjoint methods for singleDoppler wind analysis with a strong constraint of mass conservation. J. Atmos. Oceanic Technol., 11, 289-298.

_ , and _ 1995: Adjoint-method retrievals of low-altitude wind fields from single-Doppler reflectivity and radial-wind data. $J$. Atmos. Oceanic Technol., 12, 1111-1119.

_ _ _ _ and J.-X. Yu, 1994: Adjoint-method retrievals of lowaltitude wind fields from single-Doppler reflectivity measured during Phoenix-II. J. Atmos. Oceanic Technol., 11, 275-288. 